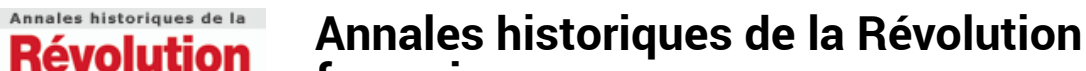 \\ française française
}

344 | avril-juin 2006

La prise de parole publique des femmes

\section{The Family on Trial in Revolutionary France}

Jacques Guilhaumou

\section{(2) OpenEdition}

\section{Journals}

Édition électronique

URL : https://journals.openedition.org/ahrf/6553

DOI : 10.4000/ahrf.6553

ISSN : 1952-403X

Éditeur :

Armand Colin, Société des études robespierristes

Édition imprimée

Date de publication : 1 juin 2006

Pagination : 239-241

ISSN : 0003-4436

\section{Référence électronique}

Jacques Guilhaumou, «The Family on Trial in Revolutionary France », Annales historiques de la

Révolution française [En ligne], 344 | avril-juin 2006, mis en ligne le 02 juillet 2008, consulté le 23 avril 2022. URL : http://journals.openedition.org/ahrf/6553 ; DOI : https://doi.org/10.4000/ahrf.6553

Ce document a été généré automatiquement le 23 avril 2022.

Tous droits réservés 


\title{
The Family on Trial in Revolutionary France
}

\author{
Jacques Guilhaumou
}

\section{RÉFÉRENCE}

Suzanne DESAN, The Family on Trial in Revolutionary France, Berkeley, Los Angeles, London, University of California Press, 2004, 456 p., ISBN 0-520-23859-1, 24.95 \$.

1 L'historienne américaine Suzanne Desan s'intéresse à la manière dont la Révolution française a transformé de manière durable la relation de la famille à la politique. Prenant en compte une grande diversité de situations - des débats à l'Assemblée nationale jusqu'aux litiges portés devant les tribunaux de famille en passant par toutes sortes d'espaces interpersonnels et interactionnels - elle s'efforce de décrire les négociations entre hommes et femmes mis en jeu au sein de la "domesticity ». Alors que Daniela Luigia Caglioti s'interroge, dans la revue Contemporanea (VIII, 2, 2005), sur les limites de l'apport habermassien en matière de réflexion sur la sphère publique féminine, Suzanne Desan montre qu'il est désormais impossible de s'en tenir à la vision de l'espace domestique en révolution comme simple espace privé, par opposition à l'espace public, mais bien au contraire qu'il convient de décrire une «action domestique», ou mieux encore "une politique domestique» partie prenante de l'espace public. Il en ressort une vision inédite de la famille comme enjeu politique dans la négociation et l'affirmation de l'individu(e) au sein de l'espace interpersonnel. Ainsi il apparait que les citoyennes peuvent prendre l'avantage dans certaines positions familiales, en négociant de nouveaux arrangements qui exigent plus de réciprocité et ouvrent des opportunités par des gains de pouvoir, de propriété, et d'indépendance. Les individu(e)s membres de la famille deviennent ainsi capables de forger des opportunités visibles au sein même de leur propre "espace domestique ", donc d'être reconnu(e)s sur le terrain politique. Nous sommes donc ici confronté à une étude exemplaire de la construction de la citoyenneté par la dynamique politique du genre. 
2 Plus concrètement, Suzanne Desan explore, textes et archives en main, les nouvelles positions sociales principalement en matière de mariage, de divorce, de réduction de l'autorité paternelle, d'héritage égalitaire entre frères et sœurs et de défense des enfants illégitimes. Elle constate ainsi la formation d'une " gender complementarity ", d'une nouvelle relation homme-femme dans l'apparition conjointe d'une référence au droit naturel et d'une idéalisation de l'affection conjugale, du moins dans les espaces de débat. La famille conjugale devient, au regard des législateurs, une "force naturelle " favorisant l'unité sociale et la complémentarité entre homme et femme. Ainsi les femmes ont charge d'activer la vitalité des hommes par leur sensibilité, leurs intuitions morales, et leur capacité à exprimer le sentiment d'humanité, ce que Desan désigne par l'expression « women's cultivation of masculine sensibility ».

3 Nous entrons, pour un temps, dans un espace révolutionnaire où règne en quelque sorte l'instabilité de genre. La forme pétitionnaire est particulièrement révélatrice d'une telle mobilité du lien féminin/masculin. D'une pétition à l'autre, les citoyennes valorisent l'attachement affectif (par exemple lorsqu'une jeune fille illégitime revendique son héritage au nom de «l'union libre » entre ses parents décédés); elles s'efforcent ainsi de valider les liens naturels, émotionnels, entre parents et enfants à l'encontre de toute forme d'exclusion patriarcale. Même si la propriété des valeurs familiales reste le fait du père, au titre de l'honneur et de la responsabilité, les qualités naturelles de la maternité sont reconnues dans leur dignité propre. Et, dans le même «mouvement domestique », la reconnaissance de la paternité met en œuvre le pouvoir «naturel» du sentiment et introduit une sensibilité masculine inédite, même si les pères cherchent avant tout à défendre leur réputation auprès de la société.

4 La contestation de l'autorité parentale devant la justice est alors appréhendée dans un cadre géographique bien délimité, la Normandie, et sur la base de cas précis. Elle révèle des formes de litige aboutissant, au titre de la revendication d'un héritage égalitaire, à des règlements d'économie morale, et induisant donc des relations de réciprocité au sein même de la famille.

5 Bien sûr, Suzanne Desan explore, dans ses derniers chapitres, le retour à l'ordre moral pendant le Directoire. La revalorisation de la puissance paternelle jugée d'utilité sociale s'opère sous la pression de pétitions « conservatrices » et suscite la perte d'une partie des avancées législatives en matière de droit égalitaire dans la relation homme-femme. Cependant, Suzanne Desan montre, que, au-delà de la rhétorique usuelle des révolutionnaires sur le rôle domestique de femmes au sens restreint, la Révolution française a enclenché un pouvoir transformateur dans la relation de genre, en multipliant les espaces domestiques de négociation au sens large, si propice à la liberté individuelle. L'acte de reconstruction sociale (" dynamic acte of social reconstruction ») qui préside au débat, puis à l'adoption du Code civil, ne détruit pas entièrement la dynamique de genre mise en place antérieurement : une nouvelle manière de penser et d'expérimenter la famille demeure, ne serait-ce qu'au titre d'une volonté maintenue d'harmoniser l'individu au tout social. Nous retrouvons ainsi la catégorie politique de famille, dont la politiste Anne Verjus s'est faite l'interprète dans son livre sur Le cens de la famille. Les femmes et le vote 1789-1848 (Belin, 2002).

6 Pour sa part, Anne Verjus développe une explication socio-historique de la mise à l'écart des femmes qui nous renvoie à une pensée politique de l'unité d'intérêts entre l'homme et la femme au sein de la famille. Le modèle politique de la famille devient, un temps, le garant de l'unité des hommes et des femmes dans le tout politique : la femme 
n'est pas exclue de la nation, elle marque son appartenance concrète à la communauté réelle des citoyens en tant que membre de la famille. Bien sûr, il persiste une différence considérable entre les sexes, ne serait-ce que par la domination du père de famille, mais cette différence n'a rien de contradictoire dans une conception de la progression de la naturalité de la société familiale à l'artificialité de la société politique. La figure fondatrice de l'individu-nation est ici assimilée au citoyen(ne) d'une famille sexuellement différenciée, socialement hiérarchisée, politiquement unie. En son sein, le père de famille, représentatif de l'autorité politique, est le garant des intérêts de tous, femmes incluses: ainsi sa capacité censitaire à devenir électeur est évaluée, si nécessaire, à partir de l'ensemble des contributions des membres, hommes ou femmes, de sa famille.

7 Suzanne Desan et Anne Verjus contribuent ainsi à promouvoir "un récit d'ouverture " sur le rôle politique des femmes pendant la Révolution française. Les citoyennes participent à part entière de l'imagination prospective et anticipatrice qui caractérise l'esprit et la pratique des révolutionnaires. Certes, non reconnues comme électrices, elles sont positionnées explicitement hors des droits politiques. Mais leur dynamique propre leur permet d'acquérir une position politique à l'intérieur même de la société politique élargie.

8 En fin de compte, l'importance de l'ouvrage de Suzanne Desan procède d'une manière originale de spécifier la dynamique de genre au contact de situations diversifiées par le recours à un vaste éventail de documents. Ainsi nous pouvons appréhender de manière particulièrement compréhensive la façon dont la Révolution, à l'initiative des citoyennes, injecte, si l'on peut dire, de la politique dans les relations interpersonnelles en termes de visibilité et de reconnaissance sociales. Précisons enfin que cet ouvrage comprend un index des personnes et des notions fort utile pour suivre telle ou telle piste de recherche, par exemple en ce qui concerne le phénomène majeur du pétitionnement. L'appareil de notes, par son importance et sa diversité, constitue également un excellent outil de travail. 